Supplement of Biogeosciences, 15, 4003-4018, 2018

https://doi.org/10.5194/bg-15-4003-2018-supplement

(C) Author(s) 2018. This work is distributed under

the Creative Commons Attribution 4.0 License.

(c) (1)

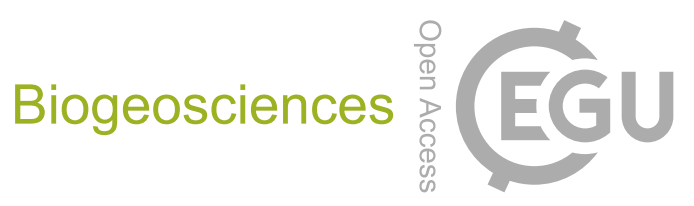

Supplement of

\title{
Inferring the effects of sink strength on plant carbon balance processes from experimental measurements
}

Kashif Mahmud et al.

Correspondence to: Kashif Mahmud (k.mahmud@westernsydney.edu.au)

The copyright of individual parts of the supplement might differ from the CC BY 4.0 License. 
Supplementary Information

Supplementary Section S1: Time dependent parameters

Supplementary Table S1: BIC values from time dependent parameter fit. The lowest BIC values indicate the best performing parameter setting. Treatment groups are: 'Small' - 5 L, 10 L and 15 L containers; 'Large' - 20 L, 25 L and 25 L containers; 'Free' - freely rooted seedlings.

Supplementary Figure S1: Total C mass in foliage NSC $\mathrm{C}_{\mathrm{n}, \mathrm{f}}$ (lines) with inferred parameter settings and corresponding observations (symbols). Note that the NSC pool (y-axes) are plotted on log scale to visualize the changes at the beginning of the experiment. Error bars (1 $\mathrm{SE}, \mathrm{n}=6$ ) are shown for each observation.

Supplementary Figure S2: Temporal evolution of carbon use efficiency (CUE) for various treatments.

\section{Supplementary material}

\section{S1. Time dependent parameters}

This section tests the time-dependency of CBM parameters $\left(k, Y, a_{\mathrm{f}}, a_{\mathrm{w}}, a_{\mathrm{r}}, s_{\mathrm{f}}\right)$ due to ontogenetic or seasonal effects. We considered two alternative parameter sets to allow this variation from the default constant parameter setup with one set of parameters, $p$ that does not change with time:

a) Linear $\left(p=p_{1}+p_{2} * \mathrm{t}\right)$ : Two sets of parameters representing linear variation over time,

b) Quadratic $\left(p=p_{1}+p_{2} * \mathrm{t}+p_{3} * \mathrm{t}^{2}\right)$ : Three sets of parameters that result in quadratic variation with time.

We examined whether parameters varied over time by comparing the BIC values for constant, linear, and quadratic parameter settings. The results are illustrated in Table S1 (Simulation Set D), which shows the effect of time dependency. Changing from constant to linear time-dependences improved BIC values for every treatment, indicating that there is significant variation over time in at least some parameters. Changing from linear to quadratic variation in parameter values also improved the goodness of fit, although to a smaller but significant extent. For example, with the optimum treatment grouping option (3 groups), BIC values indicate that the quadratic variation over time in parameters is the best option; BIC numbers are reduced by around $16 \%, 2 \%$ and $20 \%$ for small container, large container and free seedlings respectively from linear to quadratic parameter settings (Table S1). We also 
tested 3rd degree polynomial equations for parameter variation (not shown), but it increased model complexity without improving the fit.

Supplementary Table S1: BIC values from time dependent parameter fit. The lowest BIC values indicate the best performing parameter setting. Treatment groups are: 'Small' - 5 L, 10 L and 15 L containers; 'Large' - 20 L, 25 L and 25 L containers; 'Free' - freely rooted seedlings.

\begin{tabular}{|c|c|c|c|}
\hline Simulation set & Model settings & Treatment groups & $\mathrm{BIC}$ \\
\hline \multirow[t]{9}{*}{$\mathrm{D}$} & \multirow[t]{3}{*}{ Constant parameter variation } & Small & 1391 \\
\hline & & Large & 646 \\
\hline & & free & 332 \\
\hline & \multirow[t]{3}{*}{ Linear parameter variation } & Small & 826 \\
\hline & & Large & 462 \\
\hline & & free & 217 \\
\hline & \multirow[t]{3}{*}{ Quadratic parameter variation } & Small & 683 \\
\hline & & Large & 457 \\
\hline & & free & 170 \\
\hline
\end{tabular}

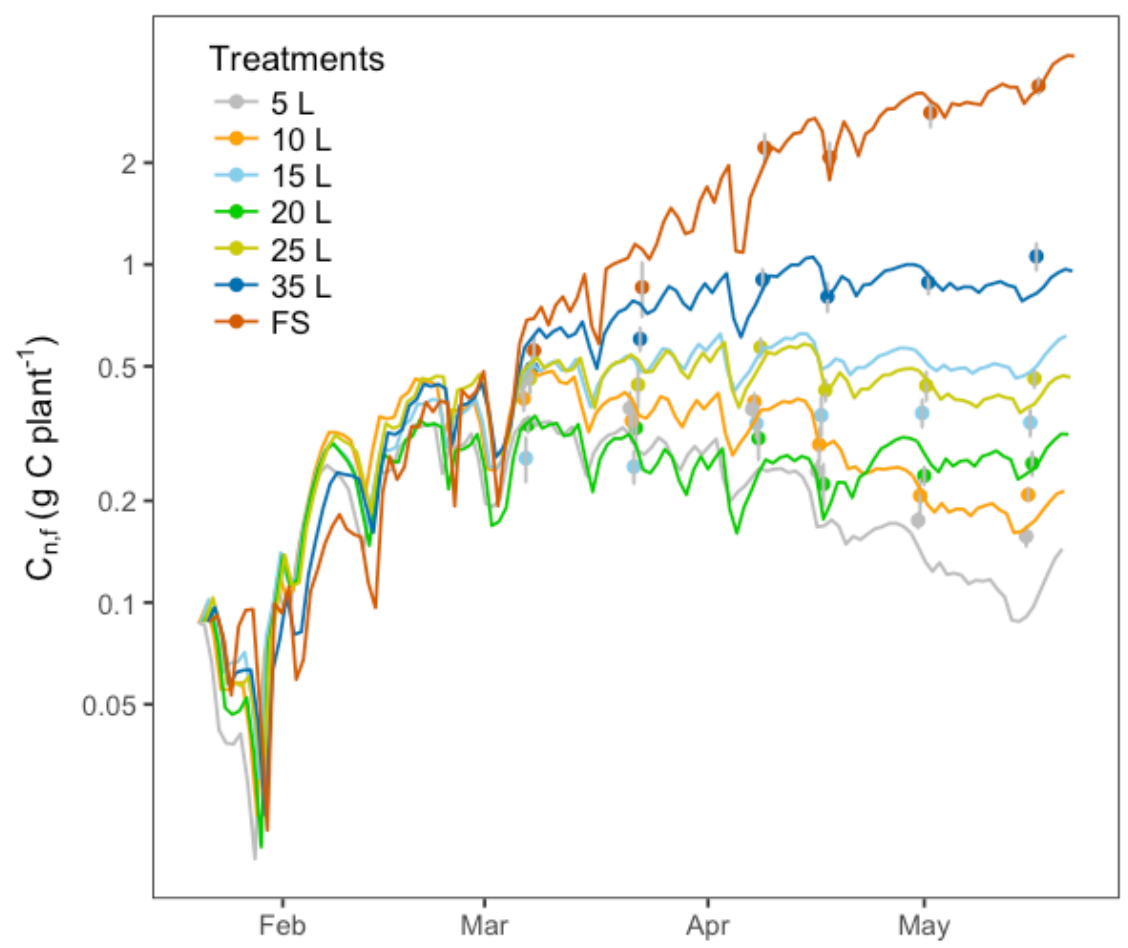

Supplementary Figure S1: Total C mass in foliage NSC $\mathrm{C}_{\mathrm{n}, \mathrm{f}}$ (lines) with inferred parameter settings and corresponding observations (symbols). Note that the NSC pool (y-axes) are 
plotted on log scale to visualize the changes at the beginning of the experiment. Error bars (1 $\mathrm{SE}, \mathrm{n}=6$ ) are shown for each observation.

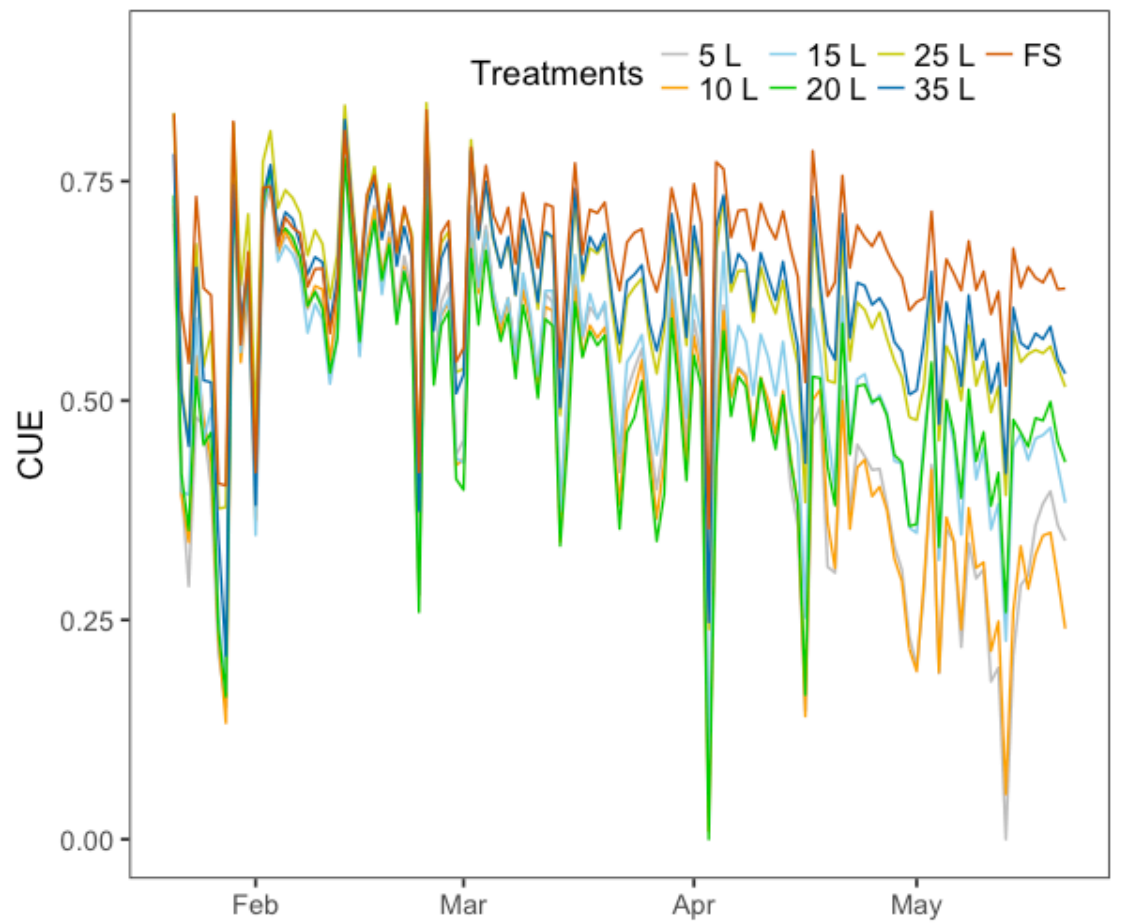

Supplementary Figure S2: Temporal evolution of carbon use efficiency (CUE) for various treatments. 